\title{
LARGE ROUGHNESS EFFECTS IN CHANNEL FLOW
}

\author{
D. M. Birch ${ }^{1}$, J. F. Morrison $^{2}$ \\ Department of Aeronautics, Imperial College, London, SW7 $2 A Z$
}

\section{INTRODUCTION}

The classical view of roughness effects, originally proposed by [1], [2], [3] and [4], assumes that local inhomogeneities arising from the specific roughness geometry are confined to a "roughness sublayer", analogous to the viscous sublayer found over smooth walls, the thickness of which is generally accepted to be about 5 roughness heights. Under these assumptions, any effects of the roughness on the turbulence away from the wall relative to the smooth-wall case must necessarily be attributable to the increase in the wall shear stress, $\tau_{w}$, alone. One can therefore expect that an overlap region of mean velocity will exist (and therefore a self-similar log law) and that the outer-region flow (or at least the mean velocity deficit and second moment) will scale exclusively with the wall friction velocity $u_{\tau}=\sqrt{\tau_{w} / \rho}$, and $h$, the outer length scale. In the present experiment, the effects of very-large roughness height $(k / h=4 \%$ and $8 \%)$ are examined. One rough surface comprises sparse and isotropic grit with a highly non-Gaussian distribution. The other is a uniform mesh consisting of twisted rectangular elements which form a diamond pattern. The mean roughness heights ( \pm the standard deviation) are, respectively, about $76 \pm 42$ and $145 \pm 150$ wall units. The mesh diamond pattern has a spanwise-to-streamwise aspect ratio of about 2.6.
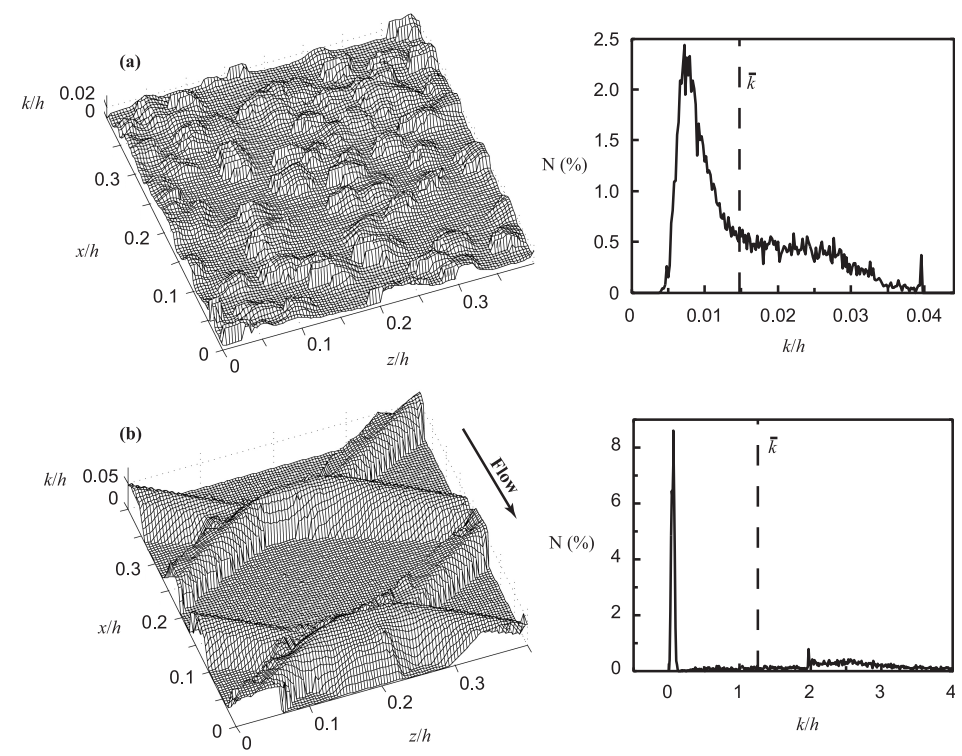

Figure 1. Surfaces: (a) grit; (b) mesh. Mean roughness height, $\bar{k}$.

\section{RESULTS}

The streamwise velocity component is measured in fully-developed turbulent channel flow for the two surfaces at comparable Reynolds numbers. The flow is shown to be two-dimensional and fully developed up to the fourth moment of velocity. Figures 2 and 3 show mean velocity profiles scaled on inner and outer variables, respectively. The roughness length, $y_{0}$, and the zero-plane displacement, $d$, are obtained by fitting data to the log law,

$$
U^{+}=\frac{1}{\kappa} \ln \left(\frac{y-d}{y_{0}}\right),
$$

\footnotetext{
${ }^{1}$ Faculty of Engineering \& Physical Sciences, University of Surrey, GU2 7XH

2j.morrison@imperial.ac.uk
} 
assuming that it begins at the wall-normal location at which the mean velocity becomes spanwisehomogeneous. $\kappa=0.41$. In the case of the mesh, measurements are performed at the centre of the diamond-patterned depression. Figure 2 suggests that, with inner scaling, the log law is apparent for both surfaces $13 \leq(y-d) / y_{0} \leq 20$. However, figure 3 suggests that self-similarity (in the form of a logarithmic law) is strictly only possible for the grit surface only, within the limited range of $0.032 \leq y / h \leq 0.05$. Figure 4 shows that the second moment scales with outer scaling $\left(u_{\tau}, h\right)$ for both surfaces.

\section{CONCLUSIONS}

At the Symposium, the distinction between self-similarity and outer similarity will be clarified and the importance of the former explained. Further results will also be presented, examining the spanwise variation of the scaling behaviour for flow over the mesh. Previous reporting of a universal behaviour of Hama's roughness function, or of an increase in the wake component over some very rough surfaces may therefore, in some cases, be erroneous and so require further investigation.

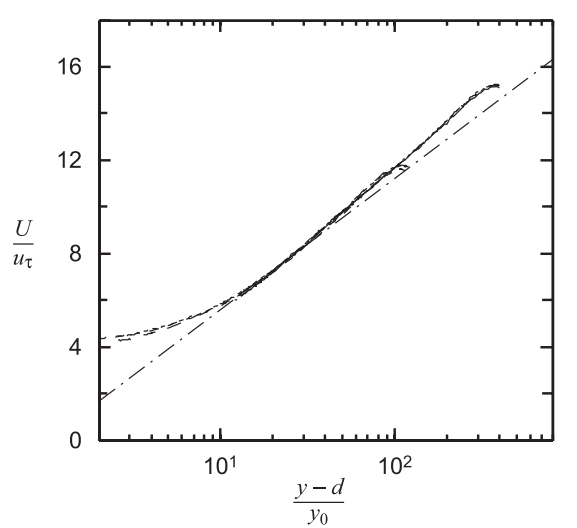

Figure 2. Mean velocity: inner scaling

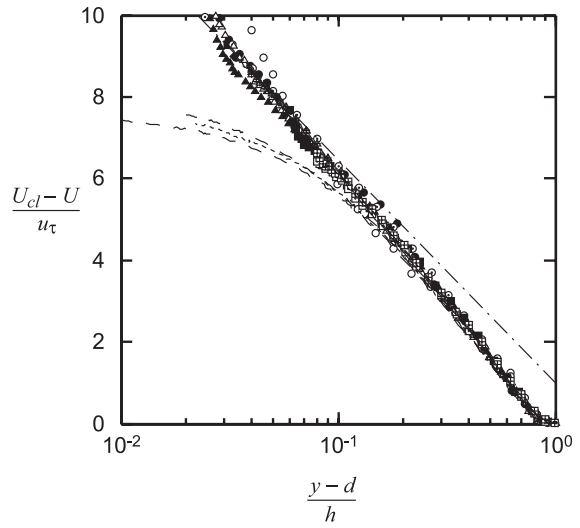

Figure 3. Mean velocity deficit: outer scaling

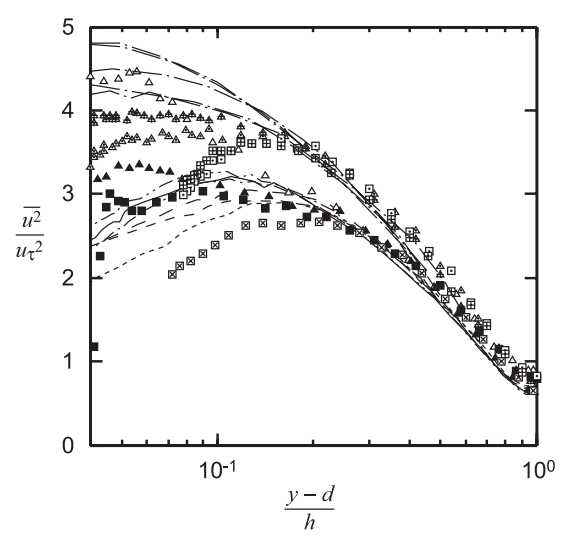

Figure 4. Second moment: outer scaling.

\section{References}

[1] F. Hama, Trans. Soc. Naval Arch. \& Marine Eng., 62, 333 (1954).

[2] F. H. Clauser, Adv. Appl. Mech., 4, 1 (1956).

[3] J. C. Rotta, Prog. Aero. Sci., 2, 1 (1962).

[4] A. A. Townsend, The Structure of Turbulent Shear Flow, Cambridge University Press, Cambridge 1976. 